\title{
Immune Activation While on Potent Antiretroviral Therapy Can Predict Subsequent CD4+ T-Cell Increases Through 15 Years of Treatment
}

\author{
Xinyan Zhang ${ }^{1}$, Peter W. Hunt ${ }^{2}$, Scott M. Hammer ${ }^{3}$, Michelle S. Cespedes ${ }^{4}$, Kristine B. \\ Patterson $^{5}$, and Ronald J. Bosch ${ }^{1}$ \\ ${ }^{1}$ Center for Biostatistics in AIDS Research, Harvard School of Public Health, Boston, \\ Massachusetts, USA \\ ${ }^{2}$ School of Medicine, University of California at San Francisco, San Francisco, California, USA \\ ${ }^{3}$ Division of Infectious Diseases, Columbia University, New York, New York, USA \\ ${ }^{4}$ School of Medicine, New York University, New York, New York, USA \\ ${ }^{5}$ Division of Infectious Diseases, University of North Carolina, Chapel Hill, North Carolina, USA
}

\begin{abstract}
Background-While persistent T-cell activation has been cross-sectionally associated with poor CD4+ T-cell restoration in HIV-infected individuals maintaining antiretroviral treatment (ART)mediated viral suppression, it remains unclear whether CD8+ T-cell activation is of predictive effect on CD4+ T-cell recovery.

Objective-We assessed whether the extent of persistent CD8+ T-cell activation (\% CD38+/ HLA-DR+) in the fi rst few years of ART-mediated viral suppression predicted subsequent CD4+ T-cell recovery in 95 subjects with up to 15 years of observation on suppressive ART.

Results-Lower CD8+ T-cell activation and higher naïve CD4+ T-cell frequencies (CD45RA+/ $\mathrm{CD} 62 \mathrm{~L}+$ ) measured at year 3 to 5 after starting ART independently predicted greater subsequent CD4+ T-cell increases. The mean CD4 count increase from year 0 to year 5 and the increase to the average of year 10 to 15 in the low CD8 activation group ( $\leq 8.5 \%$; mean $=13 \%$ ) were 342 and 458 cells $/ \mathrm{mm},{ }^{3}$ and the increases were 248 and 349 cells $/ \mathrm{mm}^{3}$ for the high CD8 activation group (>18.5\%; mean $=29 \%)(P=.002$ and $P=.016$, respectively, comparing groups). At years 10 to 15 , the mean CD4 counts in the groups were 579 and 484 cells $/ \mathrm{mm}^{3}$, respectively $(P=.026)$.
\end{abstract}

Conclusion-These fi ndings support the need to identify approaches to reduce immune activation in treated HIV disease.

\section{Keywords}

antiretroviral therapy; CD4+ T-cell counts; HIV; immune activation

(C) 2013 Thomas Land Publishers, Inc.

Address for correspondence: Xinyan Zhang, PhD, Center for Biostatistics in AIDS Research, Harvard School of Public Health, Boston, MA 02115 USA; phone: 617-432-7469; fax: 617-432-3163; xzhang@ sdac.harvard.edu.

Additional contributions: The authors thank the ACTG sites and study participants for their time and effort, Frontier Science Foundation for data management, and Summer Zheng and Eric Daar for valuable comments. We gratefully acknowledge the ALLRT protocol chairs Constance Benson and Ann Collier. 
Antiretroviral treatment (ART) substantially reduces the morbidity and mortality of human immunodeficiency virus type-1 (HIV-1)-infected patients. However, HIV-infected patients have widely variant CD4+ T-cell increases after ART, even if virally suppressed. Persistent CD8+ T-cell activation during suppressive ART has been cross-sectionally associated with poor CD4+ T-cell recovery, but no study has demonstrated that persistent T-cell activation predicts subsequent CD4 recovery in long-term studies.

For example, Hunt et al reported that persistent CD8+ T-cell activation was associated with fewer CD4+ T-cell gains from month 3 onwards in virally suppressed subjects during ART. ${ }^{1}$ Median follow-up was 21 months and the analysis was retrospective; the relationship between T-cell activation and subsequent CD4+ T-cell recovery was not assessed. High CD8+ T-cell activation was also associated with diminished concurrent CD4+ T-cell recovery during ART in another study conducted by the ACTG. ${ }^{2}$ Other studies have suggested that higher pre-ART CD8+ T-cell activation predicts poor CD4+ T-cell recovery at week $48^{3}$ or through a median of 3 years of suppressive ART. ${ }^{4}$ However, no study has definitively established that persistent CD8+ T-cell activation during ART-mediated viral suppression independently predicts subsequent $\mathrm{CD} 4+\mathrm{T}$-cell recovery. To address whether persistent CD8+ T-cell activation independently predicts subsequent CD4+ T-cell recovery during suppressive ART, we assessed the frequencies of activated CD8+ T cells and naïve CD4+ T cells 3 to 5 years after starting suppressive ART and assessed the relationships between these factors and subsequent CD4+ T-cell gains through up to 15 years of ARTmediated viral suppression.

\section{METHODS}

This investigation examined HIV-infected individuals from the ACTG $372 \mathrm{~A}$ study ${ }^{5}$ who also enrolled in the ACTG long-term observational study ALLRT. ${ }^{6}$ The subjects began protease inhibitor-based ART (indinavir +2 nucleoside reverse transcriptase inhibitors [NRTIs], zidovudine/lamivudine or stavudine/lamivudine) in 1996-1997 with CD4+ T-cell counts $\_00$ cells $/ \mathrm{mm}^{3}$ or started dual NRTI treatment with CD4+ T-cell count $\_200$ cells/ $\mathrm{mm}^{3}$ and subsequently added open-label indinavir to their dual NRTIs. ${ }^{5,7}$ At entry to the $372 \mathrm{~A}$ study, subjects were randomized to add abacavir or placebo to their indinavir-based regimen while virally suppressed $(<500$ copies $/ \mathrm{mL})$. Abacavir showed no additional effect. ${ }^{5}$

Of the 229 subjects in ACTG 372A (enrollment 1997-1998), 169 enrolled into ALLRT in 2000-2003; most (81\%) enrolled in 2000. Follow-up in 372A included HIV RNA and CD4+ T-cell count measurements every 8 weeks. These were measured every 16 weeks in ALLRT. For this investigation of long-term CD4+ T-cell count increases, the analysis was restricted to the subset of 95 subjects with HIV RNA suppression (not having 2 consecutive RNA measurements above 200 copies $/ \mathrm{mL}$ since starting indinavir-based treatment ${ }^{8}$ ) and available CD8 activation and naïve CD4 data.

CD8 activation (\%CD38+/HLA-DR+ CD8+ T cells) and naïve CD4 percentage (\% CD45RA+/CD62L+CD4+ T cells) were measured in ALLRT from 2000 to 2003 (every 16 weeks in 2000-2002; every 48 weeks in 2002-2003) using fresh cells and phenotyping at multiple labs. Immune activation and the naïve CD4 percentage were fi rst measured between 3 and 5 years after the start of indinavir-based regimen for most subjects (86\%). CD8 activation measurements during viral suppression were averaged for each subject; this was done similarly for naïve $\mathrm{CD} 4$ percentage to reduce the impact of measurement error. There was high within-subject correlation in CD8 activation $(r=0.78)$ and naïve CD4 $(r=$ 0.90) percentages based on mixed-effects models, and levels of CD8 activation and naive CD4 percentage did not change substantially over the 3 to 4 year timeframe from which the averages were constructed (mean $=2.0$ and 0.4 percentage points per year, respectively). 
Mean CD4+ T-cell counts were compared between groups (high vs low immune activation; high vs low naïve CD4 percentage) using $t$ tests. High immune activation was prespecifi ed as CD8 activation above the median and similarly high naïve CD4 percentage was defi ned as naïve CD4 percentage above the median. Mixed-effect models for longitudinal CD4+ Tcell counts from years 3 to 15 after starting indinavir-based ART were used to simultaneously assess the effect of CD8 activation and the naïve CD4 subset. To assess whether persistently high CD8+ T-cell activation could independently predict reduced CD4+ $\mathrm{T}$-cell count recovery, concurrent CD4+ T-cell counts were adjusted in another mixedeffects model. This assessment is important because persistently high CD8+ T-cell activation could conceivably be a consequence rather than a cause of a persistently low CD4+ T-cell count recovery.

Long-term increases in CD4+ T-cell counts from the start of indinavir-based treatment (year 0 ) were analyzed. Available CD4+ T-cell measurements in a 12-month window around each year were averaged for each subject for the data summarized by year. To provide a simple descriptive summary, we averaged CD4+ T-cell counts from year 10 to 15 because CD4+ Tcell counts tended to level off after year 10 and the available sample size after year 10 dropped substantially.

\section{RESULTS}

The 95 long-term suppressed subjects in this analysis were $92 \%$ male, $71 \%$ White nonHispanic, 11\% Black non-Hispanic, and 15\% Hispanic; 85 (89\%) started indinavir in combination with zidovudine and lamivudine and $10(11 \%)$ in combination with stavudine and lamivudine. At the time indinavir-based ART was initiated, the median CD4+ T-cell count was 95 cells $/ \mathrm{mm}^{3}$ (25th-75th percentiles: $37-149$ ), the median HIV RNA copies/mL level was 4.8 (25th-75th: 4.4-5.3) $\log _{10}$ (6 subjects had missing HIV RNA), and the median age was 41 (25th-75th: $35-47)$ years.

Median follow-up was 15 (25th-75th: 9-16) years. Overall, the mean CD4+ T-cell count increase from year 0 to year 5 was $296(\mathrm{SD}=150)$ cells $/ \mathrm{mm}^{3}$ and the increase from year 0 to the average of year 10 to 15 was $408(\mathrm{SD}=189)$ cells $/ \mathrm{mm}^{3}$.

The correlation between each subject's mean CD8 activation percent and mean naïve CD4 percent was $-0.24(P=.017)$. The median CD8 activation percentage between approximately years 3 to 5 of ART-mediated viral suppression was 18.5 (25th-75th: 12-27) and the median naïve CD4 percent was 32 (25th-75th: 21-41).

Figure 1a displays long-term CD4+ T-cell counts stratifi ed by each subject's average CD8 activation level. The mean CD8 activation levels in the low versus high immune activation groups were $12.8 \%$ and $30 \%$, respectively. Subjects with low CD8 activation levels on virally suppressive treatment had signifi cantly greater CD4+ T-cell count increases during follow-up, but had similar CD4+ T-cell counts at the time indinavir-based treatment was started. Specifi cally, the mean CD4+ T-cell count increase from year 0 to year 5 and the increase to the average of year 10 to 15 in the low CD8 activation group were 342 and 458 cells $/ \mathrm{mm}^{3}$, and the increases were 248 and $349 \mathrm{cells} / \mathrm{mm}^{3}$ for the high CD8 activation group ( $P=.002$ and $P=.016$, respectively, comparing groups). At years 10 to 15 , the mean CD4+ T-cell counts in the groups were 579 and 484 cells $/ \mathrm{mm}^{3}$, respectively $(P=.026)$.

Figure $1 \mathrm{~b}$ similarly summarizes long-term CD4+ T-cell counts stratifi ed by each subject's average naïve CD4 percentage. The mean naïve CD4 percentages in the 2 groups were $21 \%$ and $42 \%$, respectively. Higher naïve CD4 percentages on treatment were associated with signifi cantly greater CD4+ T-cell count increases during follow-up but similar CD4+ T-cell counts at the time indinavir-based treatment was started. Mean CD4+ T-cell count increases 
from year 0 to year 5 and years 10 to 15 were 332 and 472 cells $/ \mathrm{mm}^{3}$ in the high naïve CD4 group compared to 263 and 361 cells $/ \mathrm{mm}^{3}$ in the low naïve CD4 group $(P=.026$ and $P=$. 016, respectively, comparing groups). At years 10 to 15 , the mean CD4+ T-cell counts in the groups were 586 and 498 cells $/ \mathrm{mm}^{3}$, respectively $(P=.038)$.

CD8 activation and naïve CD4 both remained signifi cant predictors $(P<.01)$ when simultaneously evaluated in a repeated measures model. Table 1 summarizes the effect of higher CD8 activation on the continuous scale. In addition to the effects on CD4+ T-cell count at year 10 (Table 1), higher CD8 activation and lower naïve CD4 percentage were each signifi cantly associated with smaller increases in CD4+ T-cell counts from year 3 onwards in the model containing both factors $(P<.01$ for testing differences in slopes [see Table A1]). In models that included both CD8 activation and naïve CD4 percentage, neither CD4+ T-cell count nor HIV RNA level at the time indinavir-based ART was started were predictive of long-term CD4+ T-cell counts ( $P=.20$ and $P=.48$, respectively). Age, however, was a signifi cant predictor $(P=.015)$; younger subjects had greater CD4+ T-cell count increases even after adjustment for level of CD8 activation and the naïve CD4 subset on treatment. CD8 activation and naïve CD4 percentage remained as signifi cant predictors $(P<.01)$ when age was included in the model.

We further evaluated whether there was a differential effect of CD8 activation for different levels of naïve CD4 percentage. There was a non-statistically signifi cant interaction in terms of long-term CD4+ T-cell count increases $(P=.10)$. Higher CD8 activation remained associated with smaller long-term CD4+ T-cell count increases across the range of naïve CD4 percentage (Table 1). Similarly, higher naïve CD4 percentage remained positively associated with long-term CD4+ T-cell increases across the range of CD8 activation.

In sensitivity analyses, we examined the CD8 activation and naïve CD4 percentage in longterm CD4+ T-cell counts using repeated measures (generalized estimating equation [GEE]) models that require fewer assumptions than the linear mixed model. Results (data not shown) were similar to those in Table 1. Consistent fi ndings were also seen in GEE models using square-root-transformed CD4+ T-cell counts. An analysis based on CD8 activation and naïve CD4 percentage averaged over a narrower time-window also gave similar results (data not shown). In addition, a sensitivity analysis was performed restricting to subjects (and follow-up) with HIV RNA less than 50 copies/mL ( $\mathrm{n}=52$ ); fi ndings were generally similar (see Figure 1c and 1d), and both higher CD8 activation and lower naïve CD4 percentage were signifi cant predictors when simultaneously evaluated in a repeated measures model (data not shown). Last, we also found similar results (not shown) when analyzing all 169 subjects with CD8 activation data and censoring the longitudinal CD4+ Tcell counts at the time of confi rmed HIV RNA above 200 copies $/ \mathrm{mL}$.

Although both CD8 activation and naïve CD4 percentage were signifi cant predictors for CD4+ T-cell recovery, they were not predictive after adjustment for concurrent CD4+ T-cell count ( $P=.58$ and $P=.84$, respectively) in mixed-effects models. Therefore, we cannot exclude the possibility that immune activation is a consequence rather than a cause of CD4 recovery.

\section{DISCUSSION}

While persistent T-cell activation during suppressive ART has been cross-sectionally associated with poor CD4+ T-cell recovery, ${ }^{1,2,9-11}$ it has remained unclear whether persistent $\mathrm{T}$-cell activation has a predictive effect on poor CD4+ T-cell recovery in longterm studies. In the current study, we established that persistently high CD8+ T-cell 
activation and persistently low naïve CD4+ T-cell frequencies during ART-mediated viral suppression predict fewer subsequent CD4+ T-cell gains.

A key clinical question of interest is whether interventions to reduce immune activation might plausibly improve CD4+ T-cell recovery among HIV-infected individuals who have already maintained viral suppression on ART but continue to have poor CD4+ T-cell recovery. Indeed, many HIV-infected individuals experience incomplete CD4+ T-cell recovery even after a decade or more of suppressive ART, and these individuals remain at higher risk for mortality and other complications. ${ }^{12,13}$ While prior studies have established that persistently high CD8+ or CD4+ T-cell activation during ART-mediated viral suppression is cross-sectionally associated with poor CD4+ T-cell recovery, ${ }^{1-4}$ these prior studies were unable to establish whether persistent immune activation was predictive of poor CD4+ T-cell recovery since both measurements were assessed concurrently. Other studies have established that pre-ART CD8+ T-cell activation predicts subsequent CD4+ T-cell recovery during ART-mediated viral suppression. ${ }^{3,4}$ However, these latter studies only supported the hypothesis that interventions to reduce immune activation at the start of ART - such as earlier ART initiation - might plausibly improve ART-mediated CD4+ T-cell recovery. Our current study is the fi rst to establish that persistent immune activation during ART-mediated viral suppression predicts poor subsequent CD4+ T-cell recovery.

Our results are consistent with previous studies that examined CD4 counts over $1^{2,3}$ and 3 years, ${ }^{1}$ respectively. This relationship has been affi rmed through this analysis of 15 years of suppressive ART and is consistent with the recent observation that higher CD8 activation during early ART-mediated viral suppression predicts increased subsequent mortality in a Ugandan population. ${ }^{4}$

The causes of T-cell activation during ART-mediated viral suppression remain unclear, but likely include ongoing release of HIV from latently infected cells and/or low-level productive viral replication in tissue, ${ }^{14}$ persistent microbial translocation, ${ }^{15}$ and chronic viral co-infections, particularly cytomegalovirus. ${ }^{16}$ The mechanisms mediating the relationship between higher persistent immune activation and subsequent CD4+ T-cell recovery also remain unclear, but may involve suppression of hematopoietic progenitor cell proliferation, ${ }^{17}$ decreasing naïve and central memory T-cell survival through ongoing or progressive lymphoid fi brosis, ${ }^{18}$ increased CD4+ T-cell apoptosis, ${ }^{19}$ or inhibition of CD4+ T-cell proliferation. ${ }^{20}$ These potential underlying causes and mechanistic consequences of persistent immune activation may highlight targets for novel interventions to pursue in clinical trials.

While lower pre-ART naïve CD4+ T-cell frequencies have previously been shown to predict poor ART-mediated CD4+ T-cell recovery, ${ }^{2,20}$ the present work demonstrated for the fi rst time that subjects with lower naïve CD4+ T-cell frequencies during suppressive ART continue to have diminished subsequent CD4+ T-cell count increases. It remains unclear whether persistently low naïve T-cell frequencies refl ect a lack of naïve T-cell and/or hematopoietic progenitor cell production ${ }^{17}$ or decreased naïve CD4+ T-cell survival. ${ }^{18}$ Either way, both production and survival of naïve $\mathrm{T}$ cells can be increased with exogenous IL-7 administration, ${ }^{21}$ so there may be interventions to improve naïve T-cell recovery in the near future, though clinical benefi t still needs to be established.

Even though CD8 activation and naïve CD4+ T-cell frequencies were predictive of CD4+ Tcell recovery, they were not signifi cant predictors after adjustment for concurrent CD4+ Tcell counts. Causal effects of immune activation on CD4+ T-cell recovery cannot be concluded. Further investigation of the complex interplay in the infl ammatory pathways may provide signifi cant insight into the mechanisms. 
This was an observational study of virally suppressed subjects, and we did not examine comparable nonsuppressed individuals. In addition, indinavir-based regimens are no longer in common use; indeed most of our cohort had switched to non-indinavir-based regimens during follow-up. This analysis could also have bias due to informative censoring, because not all subjects were followed 15 years.

In summary, we have shown that higher levels of immune activation during virally suppressive ART are associated with reduced CD4+ T-cell count reconstitution. These fi ndings support research efforts to identify new approaches to reduce immune activation in treated HIV disease.

\section{Supplementary Material}

Refer to Web version on PubMed Central for supplementary material.

\section{Acknowledgments}

Financial support/disclosures: This study was supported in part by the AIDS Clinical Trials Group funded by the National Institute of Allergy and Infectious Diseases (AI 38858, AI 68636, AI 38855, AI 68634). S.M.H. is supported by grants AI 069470 and RR 024156.

\section{References}

1. Hunt PW, Martin JN, Sinclair E, et al. T cell activation is associated with lower CD4+ T cell gains in human immunodefi ciency virus-infected patients with sustained viral suppression during antiretroviral therapy. J Infect Dis. 2003; 187(10):1534-1543. [PubMed: 12721933]

2. Gandi RT, Spritzler J, Chan E, et al. Effect of baseline- and treatment-related factors on immunologic recovery after initiation of antiretroviral therapy in HIV-1-positive subjects: results from ACTG 384. J Acquir Immune Defi c Syndr. 2006; 42:426-434.

3. Goicoechea M, Smith DM, Liu L, May S, Tenorio AR, Ignacio CC. Determinants of CD4+ T cell recovery during suppressive antiretroviral therapy: association of immune activation, $\mathrm{T}$ cell maturation markers, and cellular HIV-1 DNA. J Infect Dis. 2006; 194:29-37. [PubMed: 16741879]

4. Hunt PW, Cao HL, Muzoora C, et al. Impact of CD8+ T-cell activation on CD4+ T-cell recovery and mortality in HIV-infected Ugandans initiating antiretroviral therapy. AIDS. 2011; 25:21232131. [PubMed: 21881481]

5. Hammer SM, Ribaudo H, Bassett R, et al. A randomized, placebo-controlled trial of abacavir intensification in HIV-1-infected adults with virologic suppression on a protease inhibitorcontaining regimen. HIV Clin Trials. 2010; 11(6):312-324. [PubMed: 21239359]

6. Smurzynski M, Collier AC, Koletar SL, et al. AIDS Clinical Trials Group Longitudinal Linked Randomized Trials (ALLRT): rationale, design, and baseline characteristics. HIV Clin Trials. 2008; 9(4):269-282. [PubMed: 18753121]

7. Hammer SM, Squires KE, Hughes MD, et al. A controlled trial of two nucleoside analogues plus indinavir in persons with human immunodeficiency virus infection and CD4 cell counts of 200 per cubic millimeter or less. AIDS Clinical Trials Group 320 Study Team. N Engl J Med. 1997; 337(11):725-733. [PubMed: 9287227]

8. Panel on Antiretroviral Guidelines for Adults and Adolescents. Guidelines for the use of antiretroviral agents in HIV-1-infected adults and adolescents. Department of Health and Human Services; http://www.aidsinfo.nih.gov/contentfiles/lvguidelines/adultandadolescentgl.pdf

9. Massanella M, Negredo E, Pérez-Alvarez N, et al. CD4 T-cell hyperactivation and susceptibility to cell death determine poor CD4 T-cell recovery during suppressive HAART. AIDS. 2010; 24(7): 959-968. [PubMed: 20177358]

10. Solomon A, Cameron PU, Bailey M, et al. Immunological and virological failure after antiretroviral therapy is associated with enhanced peripheral and thymic pathogenicity. $\mathrm{J}$ Infect Dis. 2003; 187(12):1915-1923. [PubMed: 12792868] 
11. Onen NF, Overton ET, Presti R, Blair C, Powderly WG, Mondy K. Sub-optimal CD4 recovery on long-term suppressive highly active antiretroviral therapy is associated with favourable outcome. HIV Med. 2009; 10(7):439-446. [PubMed: 19459993]

12. Kelley CF, Kitchen CM, Hunt PW, et al. Incomplete peripheral CD4+ cell count restoration in HIV-infected patients receiving long-term antiretroviral treatment. Clin Infect Dis. 2009; 48(6): 787-794. [PubMed: 19193107]

13. Baker JV, Peng G, Rapkin J, et al. Terry Beirn Community Programs for Clinical Research on AIDS (CPCRA). CD4+ count and risk of non-AIDS diseases following initial treatment for HIV infection. AIDS. 2008; 22(7):841-848. [PubMed: 18427202]

14. Yukl SA, Shergill AK, McQuaid K, et al. Effect of raltegravir-containing intensifi cation on HIV burden and T-cell activation in multiple gut sites of HIV-positive adults on suppressive antiretroviral therapy. AIDS. 2010; 24(16):2451-2460. [PubMed: 20827162]

15. Jiang W, Lederman MM, Hunt PW, et al. Plasma levels of bacterial DNA correlate with immune activation and the magnitude of immune restoration in persons with antiretroviral-treated HIV infection. J Infect Dis. 2009; 199(8):1177-1185. [PubMed: 19265479]

16. Hunt PW, Martin JN, Sinclair E, et al. Valganciclovir reduces T cell activation in HIV-infected individuals with incomplete CD4+ T cell recovery on antiretroviral therapy. J Infect Dis. 2011; 203(10):1474-1483. [PubMed: 21502083]

17. Sauce D, Larsen M, Fastenackels S, et al. HIV disease progression despite suppression of viral replication is associated with exhaustion of lymphopoiesis. Blood. 2011; 117(19):5142-5151. [PubMed: 21436070]

18. Zeng M, Smith AJ, Wietgrefe SW, et al. Cumulative mechanisms of lymphoid tissue fi brosis and T cell depletion in HIV-1 and SIV infections. J Clin Invest. 2011; 121(3):998-1008. [PubMed: 21393864]

19. Herbeuval JP, Grivel JC, Boasso A, et al. CD4+ T-cell death induced by infectious and noninfectious HIV-1: role of type 1 interferon-dependent, TRAIL/DR5-mediated apoptosis. Blood. 2005; 106(10):3524-3531. [PubMed: 16046522]

20. Notermans DW, Pakker NG, Hamann D. Immune reconstitution after 2 years of successful potent antiretroviral therapy in previously untreated human immunodefi ciency virus type 1-infected adults. J Infect Dis. 1999; 180:1050-1056. [PubMed: 10479130]

21. Sereti I, Dunham RM, Spritzler J, et al. IL-7 administration drives T cell-cycle entry and expansion in HIV-1 infection. Blood. 2009; 113:6304-6314. [PubMed: 19380868]

\section{APPENDIX}

\section{Table A1}

Effect of CD8 activation (\% CD38+/HLA-DR+) and naïve CD4 percentage (\% CD45RA+/ $\mathrm{CD} 62 \mathrm{~L}+)$ on the slope of change in CD4 outcome in virally suppressed subjects

\begin{tabular}{lcc}
\hline & \multicolumn{2}{c}{ Effect on mean CD4 count per year (95\% CI) } \\
\cline { 2 - 3 } Predictor & Separate univariate models & $\begin{array}{c}\text { Model with both CD8 activation and } \\
\text { naïve CD4 percentage }\end{array}$ \\
\hline $\begin{array}{l}\text { CD8 activation } \\
\text { points higher) }\end{array}$ & $-3(-5,-1)$ & $-3(-5,-1)$ \\
$\begin{array}{l}\text { Naïve CD4 } \%^{a} \text { (per 5 percentage points } \\
\text { higher) }\end{array}$ & $3(1,4)$ & $2(1,-4)$ \\
\hline a Each subject's average level, measured primarily during years 3-5, was used as the predictor.
\end{tabular}


(a)

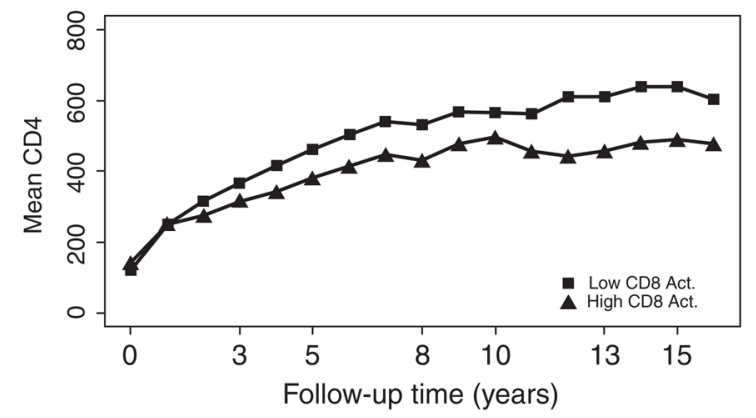

$\begin{array}{llllllll}\text { Low CD8 Act. } & 48 & 48 & 48 & 42 & 38 & 25 & 25 \\ \text { High CD8 Act. } & 47 & 47 & 47 & 37 & 31 & 27 & 25\end{array}$

(c)

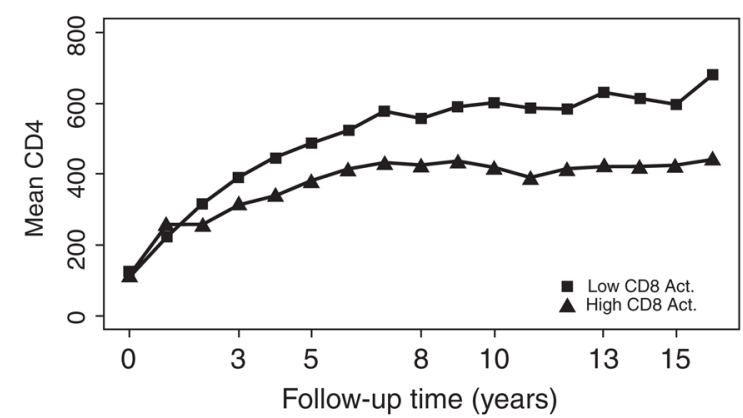

$\begin{array}{clllllll}N & & & & & & 15 \\ \text { Low CD8 Act. } & 26 & 26 & 26 & 23 & 19 & 15 \\ \text { High CD8 Act. } & 26 & 26 & 26 & 21 & 15 & 14 & 13\end{array}$ (b)

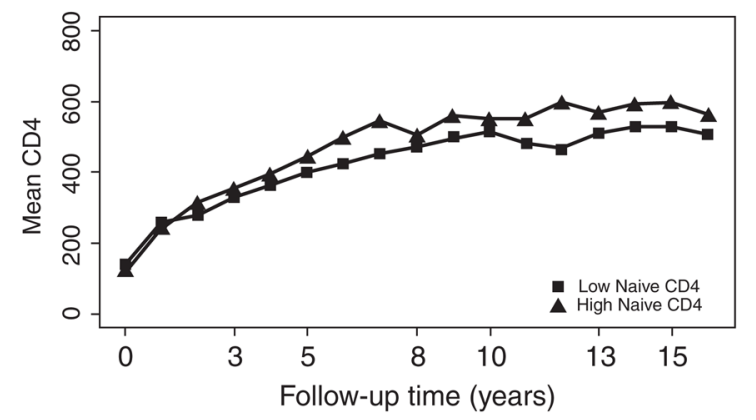

$\begin{array}{rrrrrrrr}\text { N } & & & & & & \\ \text { High Naive CD4 } & 47 & 47 & 47 & 38 & 32 & 23 & 22 \\ \text { Low Naive CD4 } & 48 & 48 & 48 & 41 & 37 & 29 & 29\end{array}$

(d)

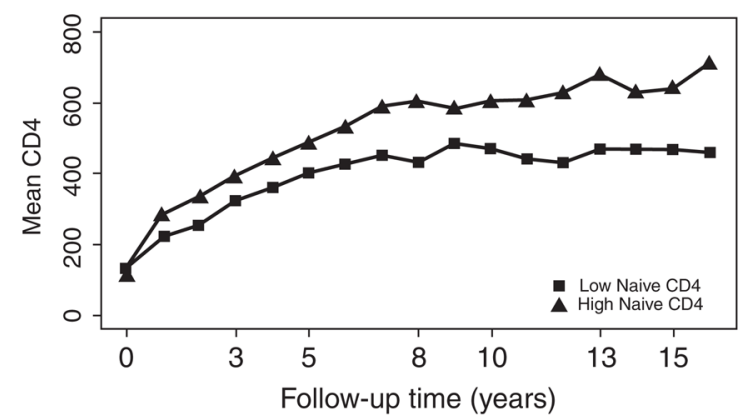

$\begin{array}{cccccccc}N & & & & & & & \\ \text { High Naive CD4 } & 26 & 26 & 26 & 21 & 15 & 12 & 11 \\ \text { Low Naive CD4 } & 26 & 26 & 26 & 23 & 19 & 17 & 17\end{array}$

Figure 1.

Mean CD4+ T-cell counts over time in virally suppressed subjects, using 200 copies $/ \mathrm{mL}$ virologic failure threshold for panels (a) and (b) and using a 50 copies $/ \mathrm{mL}$ threshold for panels (c) and (d), stratifi ed by (a) high versus low (>18.5\% vs $\leq 8.5 \%$ ) CD8+ T-cell activation (\% CD38+/HLA-DR), (b) high versus low ( $<32 \%$ vs $\$ 32 \%$ ) naïve CD4+ T-cell perentage (\% CD45RA+/CD62L+), (c) high versus low (>18.9\% vs $₫ 8.9 \%$ ) CD8+ T-cell activation (\% CD38+/HLA-DR), (d) high versus low (>33\% vs $₫ 3 \%$ ) naïve CD4+ T-cell percentage (\% CD45RA+/CD62L+). CD8+ T-cell activation and naïve CD4+ T-cell percentage were averaged levels measured primarily during years 3-5. 
Table 1

Effect of CD8 activation (\% CD38+/HLA-DR+) and naïve CD4 percentage (\% CD45RA+/CD62L+) on longterm CD4 outcomes in virally suppressed subjects

\begin{tabular}{|c|c|c|c|c|}
\hline \multirow{4}{*}{$\begin{array}{l}\text { Predictor } \\
\text { CD8 activation }{ }^{a} \text { (per } 5 \text { percentage } \\
\text { points higher) }\end{array}$} & \multicolumn{4}{|c|}{ Effect on mean CD4 count at year $10(95 \%$ CI) } \\
\hline & \multirow{2}{*}{$\begin{array}{c}\text { Separate univariate models } \\
-34(-51,-18)\end{array}$} & \multirow{2}{*}{$\begin{array}{c}\begin{array}{c}\text { Model with both CD8 } \\
\text { activation and naïve } \\
\text { CD4 percentage }\end{array} \\
-28(-44,-12) \\
\end{array}$} & \multicolumn{2}{|c|}{ Model with both factors and interaction } \\
\hline & & & Naïve $\mathrm{CD} 4 \%=21 \%$ & $-30(-48,-13)$ \\
\hline & & & Naïve CD4\%=32\% & $-28(-44,-12)$ \\
\hline & & & Naïve $C D 4 \%=41 \%$ & $-26(-44,-8)$ \\
\hline \multirow{3}{*}{$\begin{array}{l}\text { Naïve CD } 4 \%^{a} \text { (per } 5 \text { percentage } \\
\text { points higher) }\end{array}$} & $28(16,41)$ & $24(12,36)$ & $\mathrm{CD} 8$ activation $=12 \%$ & $22(7,37)$ \\
\hline & & & CD8 activation $=18.5 \%$ & $23(10,36)$ \\
\hline & & & CD8 activation $=27 \%$ & $25(12,38)$ \\
\hline
\end{tabular}

[ach subject's average level, measured primarily during years 3-5, was used as the predictor. 